\title{
Entrée dans la profession de traducteur-interprète
}

\author{
Félicien Tcham Ewane
}

\section{OpenEdition}

Journals

Édition électronique

URL : http://journals.openedition.org/esp/1135

DOI : 10.4000/esp. 1135

ISSN : 2532-0319

\section{Éditeur}

Centre d'Information sur l'Éducation Bilingue et Plurilingue

Édition imprimée

Date de publication : 1 juin 2017

Pagination : 79-86

ISSN : 1127-266X

\section{Référence électronique}

Félicien Tcham Ewane, «Entrée dans la profession de traducteur-interprète », Éducation et sociétés plurilingues [En ligne], 42 | 2017, mis en ligne le 01 février 2018, consulté le 15 septembre 2020. URL: http://journals.openedition.org/esp/1135 


\section{ENTRÉE DANS LA PROFESSION DE TRADUCTEUR-INTERPRÈTE}

\section{Félicien Tcham EWANE}

Itinerario di un giovane Camerunese che, cresciuto in due famiglie, due culture, due dialetti, parla francese fin dalla scuola materna e si trova esposto fin dalla scuola primaria alla lingua inglese, sceglie poi di imparare l'italiano al Centro italiano di cultura della capitale de Camerun e sviluppa l'ambizione di impegnarsi nello sviluppo delle relazioni bilaterali fra il Camerun e l'Italia. Nasce cosi una vocazione di traduttore-interprete fra francese ed italiano.

Parole chiave: Camerun, dialetti, francese, italiano, vocazione, traduttore, interprete

The article describes the itinerary of a young man from Cameroon, raised by two families, in two cultures and two dialects, who spoke French as of age 3 and learned English in primary school. He then chose to study Italian at the Italian Cultural Center in Yaoundé with the aim of participating in the development of relations between Cameroon and Italy by becoming a translator-interpreter in French and Italian.

Key-words: Cameroon, dialects, French, Italian, vocation, translator, interpreter

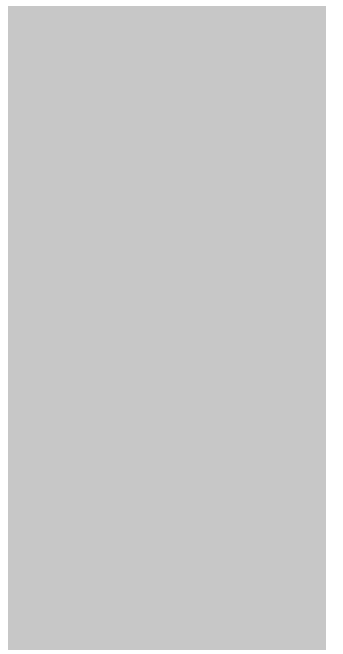

'exprimer sur ma propre expérience du bilinguisme et du plurilinguisme, c'est définir avant tout mes origines traversées grâce à la volonté et l'engagement.

La date du 21 février est une date importante pour le Cameroun, une nation qui contient environ 250 dialectes; ce jour-là on célèbre la «Journée internationale des langues maternelles».

Ma langue maternelle est le ghômala, que je parle couramment parce que j'ai été éduqué par ma famille maternelle. J'ai appris le français au Cameroun pendant mes années d'études primaires et secondaires.

Né en 1985 alors que la maman était encore collégienne mineure et vivait chez ses parents. Cette grossesse trouble la famille, où les frères aînés n’apprécient pas que leur jeune sœur se trouve dans cet état. Mais l'autorité du père de ma mère, enseignant dans une école catholique, met de l'ordre et s'occupe de tout, au point d'être à l'origine de mon futur projet de poursuivre mes études 


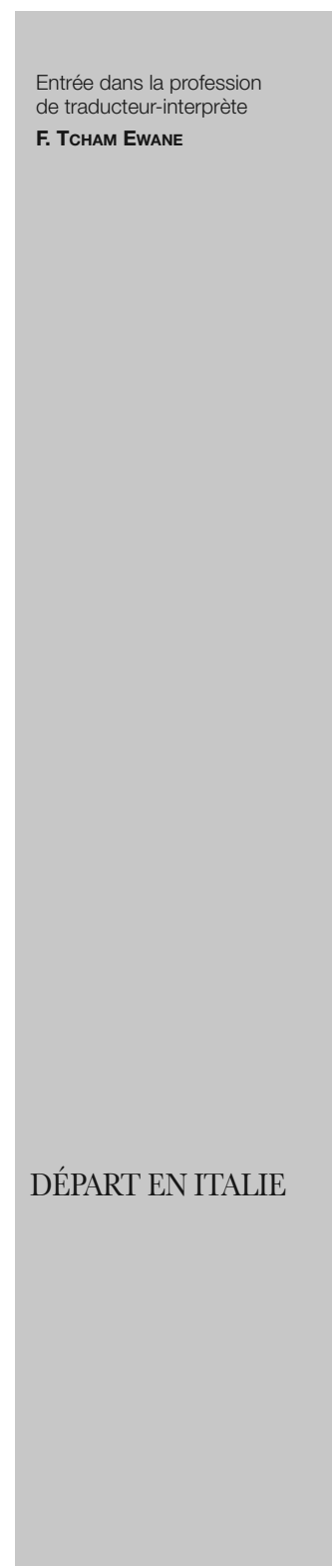

en Italie. A ma connaissance, mon grand-père n'avait pas de lien particulier avec l'Italie ou avec la langue italienne mais je crois qu'il avait la volonté de soutenir mes projets.

Pendant cette grossesse, mon père prendra la fuite. Ma carte de baptême à la Paroisse Notre Dame des Apôtres de Loum-chantiers porte mon nom Tcham Félicien. Le nom de mon père Ewane sera intégré dans mon acte de naissance seulement lorsque je présenterai mon examen national, à savoir le Certificat d'études primaires.

Ma famille maternelle est de l'Ouest: Bayangam. Ma famille paternelle est du Littoral: Mbo.

Deux familles, deux cultures, deux dialectes.

Après l'école maternelle à Loum chez mes grands-parents, je suis envoyé chez la sœur aînée de ma mère qui est mariée à Douala. Ce changement me trouble et je souhaite rentrer à Loum, mais par la suite je m'adapte.

Comme tout jeune Camerounais, dès l'école primaire je reçois des cours de langue anglaise.

J'ai passé des vacances d'une durée de trois mois chez ma grandmère paternelle à Kumba qui est une ville de la région du SudOuest, une des deux régions anglophones que compte le Cameroun. Dans cette ville de Kumba, j'ai appris quelques notions du pidgin English, l'anglais local très influencé par la proximité géographique du Nigéria.

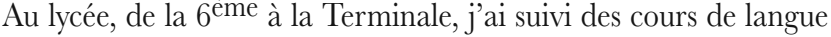
anglaise.

Après le Baccalauréat, je suis parti à Yaoundé suivre des cours de langue italienne au Centre culturel italien. Je retiendrai une astuce qui se révèlera fructueuse. En effet, nos enseignants nous disaient très souvent que nous ferions d'énormes progrès lorsque nous arrêterions de penser en français.

Je suis allé en Italie parce que j'avais été impressionné par la Coupe du monde de football de 1990 qui s'est tenue en Italie et par la bonne performance des «Lions Indomptables» (équipe nationale du Cameroun). J'étais curieux de découvrir ce pays, cela faisait partie de mes rêves. La seconde raison de mon départ est d'ordre religieux: mon appartenance à l'église catholique. Mon départ en Italie nécessitait le dépôt d'une caution bancaire d'une valeur égale à 4000 Euros environ (contribution familiale) dont le reçu devait être remis à l'ambassade d'Italie au Cameroun. 


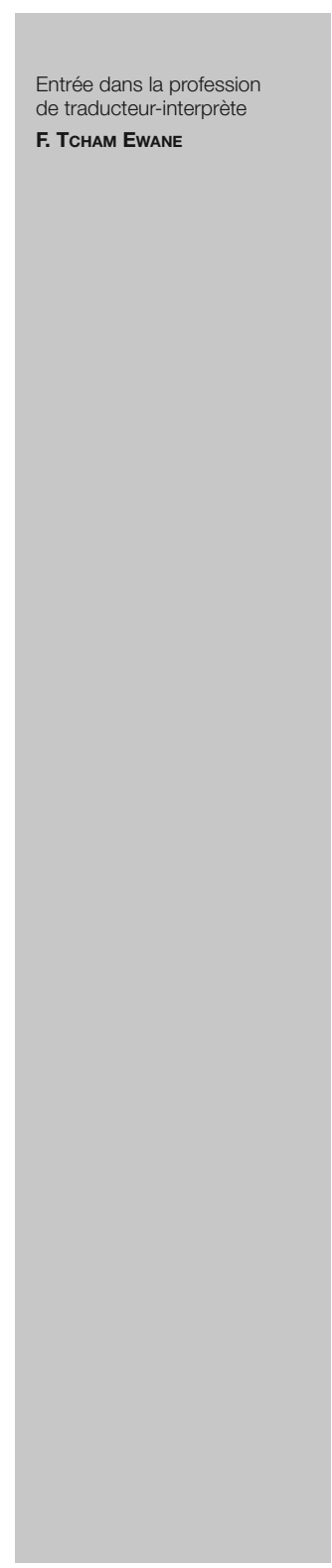

En Italie, j'ai obtenu une bourse de l'Azienda regionale per il diritto agli studi superiori (Arestud, aujourd'hui Er.Go).

Lorsque j'arrive en Italie en 2006, je prends conscience que mon niveau en langue italienne est bas. Je ne suis pas capable de m'exprimer avec aisance dans toutes les circonstances de la vie: l'administration, les chantiers de construction, l'économie, les finances, la jurisprudence, la médecine...

Ce constat me pousse à combler mes lacunes. Je procède par étapes et de manière méthodique. Déjà, je souhaitais m’impliquer à l'avenir dans le développement des relations diplomatiques et bilatérales entre le Cameroun et l'Italie.

En Italie, je lisais beaucoup de livres et je regardais la télévision. J'achetais des journaux tels que L'Osservatore Romano, La Repubblica, La Stampa. J'achetais aussi des magazines de gymnastique mentale: mots croisés, devinettes, chercher le mot.

Cette étape une fois passée, mon souci était de vérifier si je pouvais traduire un texte italien en langue française et aussi traduire un texte français en langue italienne, en me servant de mon dictionnaire Larousse français-italien que j'utilisais au Cameroun lorsque je suivais les cours d'italien à Yaoundé.

Il est important de souligner qu'à l'époque des préparations aux examens écrit et oral pour l'obtention du Certificato di conoscenza della lingua italiana Celi 3 , je m'amusais à parcourir toutes les pages de mon dictionnaire de poche Larousse. Cet exercice me sera très bénéfique lorsque je m'impliquerai dans des traductions de textes qui s'étendent sur des dizaines de pages.

Lorsque j'avais vérifié que je pouvais traduire un texte sans avoir recours à mon dictionnaire de poche Larousse qui était toujours sur ma table d'étude, j'ai compris que je pouvais encore progresser, afin de traduire de manière instantanée la conversation entre un français et un italien.

Mais je rencontre un obstacle. Je n'ai pas trouvé un environnement propice pour mettre à l'épreuve mes qualités de futur interprète.

En 2010, je fais un stage de trois mois à Leipzig en République Fédérale d'Allemagne dans le cadre du programme européen Leonard De Vinci, le Lifelong Learning Program. J'avais découvert ce programme dans un journal et constitué un dossier qui avait été retenu. Je redécouvre ainsi la langue allemande que j'avais étudiée au lycée, en 4è et en 3è.

Je reçois un signal fort lors de l'examen oral de l'option B (électronique et communications). Le professeur qui pensait que j'étais bloqué parce que je ne trouvais pas les mots en langue italienne, 


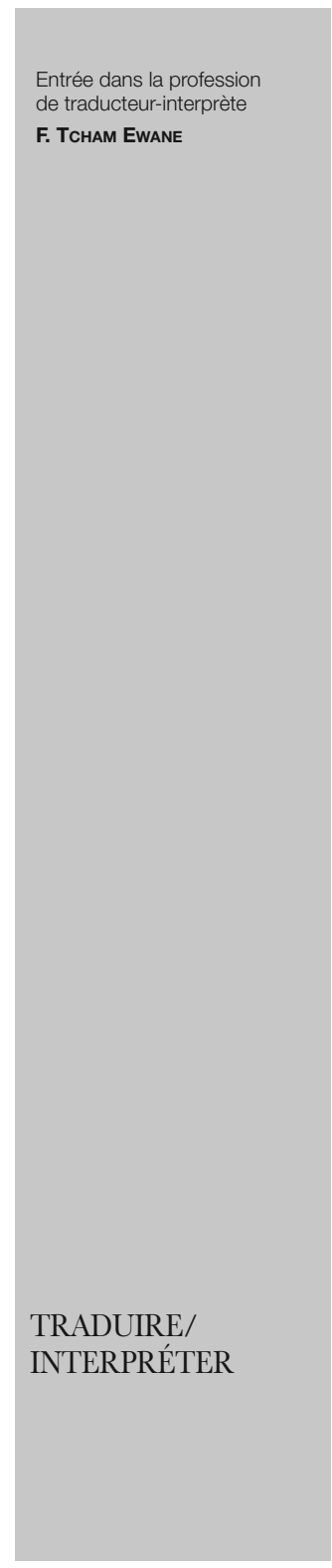

m'invita à poursuivre en langue française. Malgré cette remarque du professeur, j'ai continué à m'exprimer en langue italienne. A la fin de cet examen oral, il m'a parlé en français certainement pour s'assurer que je parle français.

Ma première expérience d'interprète a lieu lorsque je suis admis à la prestigieuse Fondazione Collegio San Carlo di Modena. A la fin d'un séminaire sur les vertus des médicaments, le directeur du Collège me demande - mais sans me préparer avant - de présenter notre Fondation et ses activités de formation au professeur invité, en langue française.

J'étais bloqué par l'improvisation du directeur et les émotions ne m'ont pas aidé à rester calme. Je constatais que je ne parvenais pas à articuler mes phrases. J'étais très bouleversé parce que je pensais que le directeur devait me voir négativement. En plus, je suis censé parler couramment français parce que c'est ma première langue structurée.

Qu'est-ce qui s'était passé? Cette semaine-là, j'étais troublé. Je comprendrai quelques mois plus tard que je n'avais aucun problème.

C'est la méthode qui n'avait pas été bonne. L'approche aurait voulu que je sois avisé quelques heures avant, afin de me préparer à recevoir ce professeur français pour lui présenter la Fondation, centre de formation et d'excellence tant au niveau national qu'international.

Après cet épisode, le directeur du Collège de la Fondation Don Luca Balugani, prêtre de l'Archidiocèse de Modène, qui avait décelé une certaine vocation dans mes comportements, décida de m'envoyer en paroisse pour une période de discernement. La paroisse de Gesù Redentore in via Leonardo da Vinci, où j’ai résidé jusqu'à mon retour au Cameroun, était devenu mon lieu de vie et de prière. Le curé de cette paroisse organisait des concerts d'orgue. Des artistes célèbres comme Jean Guillou y venaient. Je serai invité à faire découvrir la ville de Modène à l'un d'entre eux.

Afin de m'entraîner, j'ai aussi commencé à traduire des documents spécialisés d'économie et d'ingénierie.

Lorsque je rentre au Cameroun, je développe davantage mes talents de traducteur. Récemment, un Centre de promotion de la langue italienne m'a sollicité pour devenir leur traducteur en vue de délivrer des traductions officielles d'actes de naissance, diplômes, etc.

Pour cela, je dois passer un examen à l'Ambassade d'Italie qui m'a envoyé un document en langue italienne concernant les 


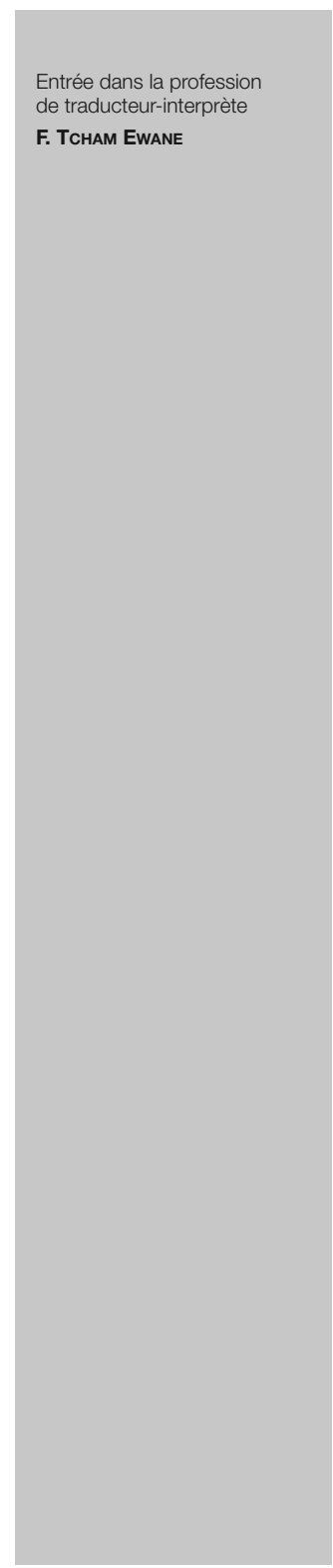

conseils et les informations pour les aspirants traducteurs. Je l'ai traduit en langue française:

«L'aspirant traducteur doit envoyer un message à culturale.yaounde@esteri.it afin de demander un rendez-vous pour subir l'examen. L'ambassade fixera une date, à sa discrétion, de telle manière qu'elle soit compatible avec ses propres engagements de travail. Le fait de savoir bien parler et bien écrire une langue ne fait pas nécessairement de l'intéressé un traducteur. La traduction est un art difficile, à travers lequel on doit exprimer des idées, des concepts et des faits, pour les translater d'une langue (source) à une autre (cible), sans déformer le contenu originel et en s'efforçant de l'exprimer de manière correcte, appropriée et compréhensible. Il est donc indispensable que le traducteur maîtrise parfaitement au moins deux langues, tant du point de vue de la grammaire et de la syntaxe, cela est valable pour toute la série de subtilités, double-sens, proverbes, difficultés intrinsèques propre à toute langue, que du point de vue culturel, étant donné que, seuls ceux qui ont longtemps étudié et approfondi certaines expressions sont capables de les saisir et donc de les exprimer de manière correcte. Le traducteur doit savoir «traduire» sans nécessairement «interpréter», sauf dans les cas où on ne peut faire autrement. Par conséquent, seule la pratique constante et régulière permettra au traducteur de savoir quand il doit se limiter à traduire et quand il doit interpréter. En d'autres termes, c'est avec l'exercice constant de la traduction et de la lecture que le traducteur sait quand il faut écrire des phrases en employant des termes qui, bien que ne reflétant pas la traduction littérale du texte originel, sont sans doute plus appropriés pour des raisons de clarté. Il est opportun d'attirer l'attention de celui qui s'intéresse à la traduction sur la nécessité de vérifier attentivement ce qu'il écrit et de s'assurer qu'il ait un sens dans la langue cible. En effet, très souvent, la traduction littérale d'une phrase ou d'un terme n'a aucun sens dans la langue cible. Pour s'exercer à l'art de la traduction, il est important de travailler sur des textes tels que les verdicts du Tribunal, les contrats de travail, les accords commerciaux, etc. pour lesquels on ne peut se limiter à une traduction littérale: ces textes exigent non seulement une connaissance approfondie des styles de rédaction, mais aussi la parfaite maitrise des termes juridiques. Enfin, lorsqu'on travaille sur une traduction, il faut au minimum trois dictionnaires: le dictionnaire bilingue et d'autres dictionnaires (avec les définitions) tant dans la langue initiale que dans la langue finale. On conseille vivement d'acheter le dictionnaire bilingue des termes juridiques et économiques.» 


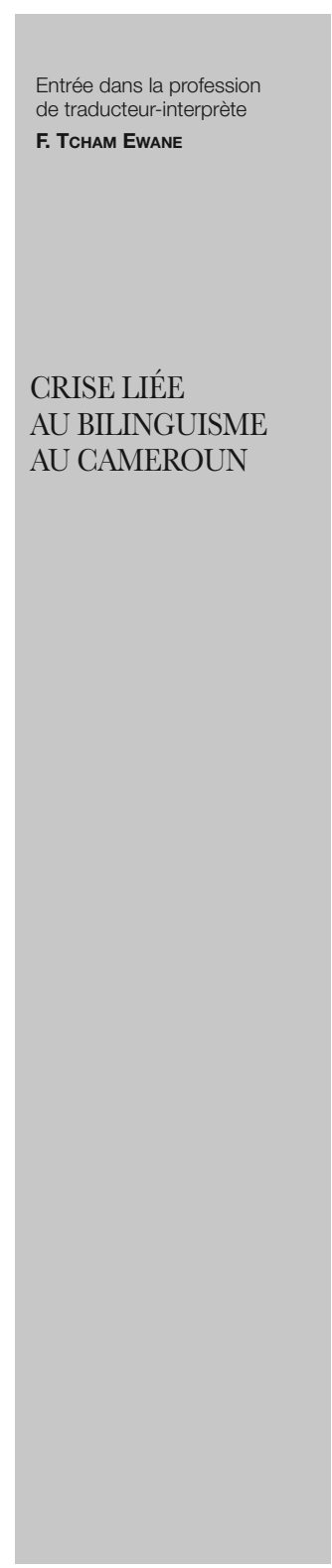

Une crise se produit au Cameroun qui met en lumière l'importance de la traduction qui reste une discipline méconnue. C'est cette crise qui vient révéler à l'opinion publique camerounaise le bien-fondé de la traduction.

Elle me concerne personnellement parce que primo, je suis un citoyen camerounais qui milite pour la paix au Cameroun et pour l'unité dans la diversité culturelle qui caractérise le Cameroun, et secondo, cette crise interpelle ma réflexion dans la mesure où je constate que le traducteur-interprète est un médiateur qui permet à travers le dialogue, la négociation et la concertation à deux entités de langues différentes de se faire comprendre en cas de litiges, de se rapprocher en cas de conflits, et in fine, de trouver des solutions de paix durable.

Le Cameroun, pays qui compte huit régions francophones et deux régions anglophones, vient donc d'éviter de justesse une crise liée au bilinguisme. Il est devenu indépendant, uni et bilingue en 1960. Les anglophones estiment qu'ils font beaucoup d'efforts pour s'exprimer en français, et à l'inverse, que les francophones ne s'efforcent pas de s'exprimer en anglais. En outre, les anglophones déclarent qu'ils ne sont pas assez entendus, compris et représentés dans les affaires de l'Etat.

En décembre 2016, le malaise commence à envahir les enseignants anglophones et les avocats anglophones qui exigent la traduction des textes en langue anglaise. Les avocats anglophones vont se mobiliser pour que leur voix soit entendue. Malheureusement, certains groupes récupéreront ce malaise des avocats pour en faire leur problème. C'est ainsi que toutes les couches sociales s'ingèrent, s'impliquent et en janvier 2017, décident de faire grève. On assiste à des actes de délinquance dans les régions du Sud-Ouest et du Nord-Ouest. L'école est interdite. Les universités sont vides, les lycéens sont à la maison. L'Etat cherche à résoudre ce problème qui est compliqué. Les ministres de la justice, de l'enseignement supérieur, de l'enseignement secondaire, de la communication, de l'administration territoriale réagissent dans les médias, se concertent avec la société civile, cherchent des solutions de sortie de crise. Des comités interministériels se créent pour étudier le phénomène. Les services du Premier Ministre s'activent pour recueillir les revendications et apporter des solutions.

Les avocats anglophones des régions du Nord-Ouest et du SudOuest réclamaient, entre autres, la traduction en anglais des Actes Uniformes OHADA (Organisation pour l'Harmonisation en Afrique du Droit des Affaires). Les textes OHADA sont élaborés à 


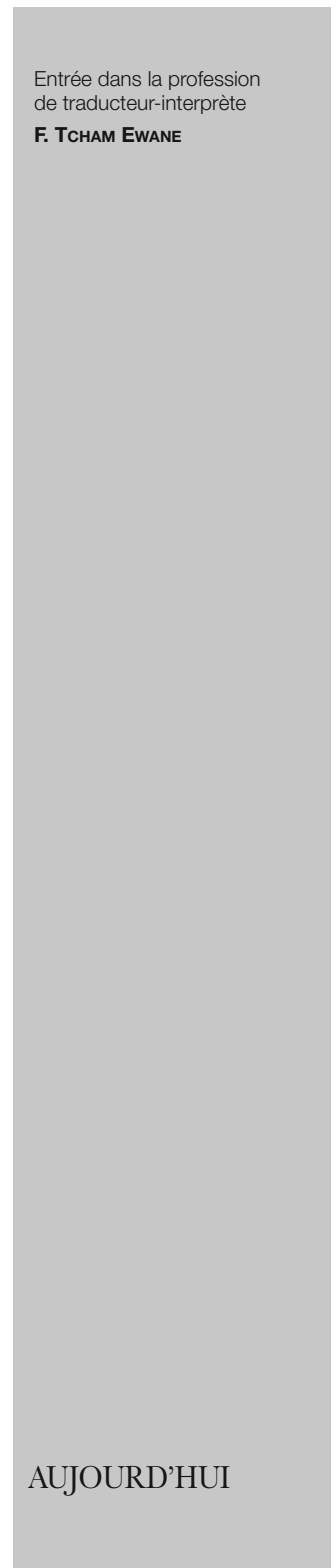

partir d'instruments juridiques internationaux qui prennent en compte tous les systèmes juridiques et économiques en vigueur dans le monde. Le Ministère de la Justice de la République du Cameroun a entrepris une démarche particulière auprès du Secrétariat Permanent de l'OHADA, pour que soit modifiée la disposition du Traité qui instituait le français comme seule langue de travail de l'OHADA. C'est ainsi qu'à la demande du Cameroun, le Traité de Québec a modifié l'article 42 du Traité OHADA pour faire dorénavant du français, de l'anglais, de l'espagnol et du portugais, les langues de travail de l'OHADA.

En 2008, lorsque le Traité de Québec a modifié celui de Port Louis en instituant l'anglais comme l'une des langues de travail de l'OHADA, le Secrétariat Permanent a souhaité que la publication de la version anglaise de ces Textes soit au préalable approuvée par le Ministère de la Justice du Cameroun. Le Ministère de la Justice a donc mis en place un Comité composé de hauts magistrats de la Cour Suprême, des Chefs des Cours d'Appel, ainsi que d'autres magistrats en service à la Chancellerie, pour la plupart anglophones, pour revisiter les textes en anglais. Mais, entre-temps, le Conseil des Ministres de l'OHADA a engagé l'actualisation de certains Actes Uniformes. Ce qui a eu pour effet de ralentir les travaux de relecture engagés.

La publication des Actes Uniformes de l'OHADA en anglais dans le Journal Officiel de l'OHADA le 24 novembre 2016 dernier, est venue mettre un terme à ce long processus débuté depuis plusieurs années. Le document produit par le Secrétariat Permanent a près de 600 pages d'un travail scientifique et technique de haut niveau. Dans un communiqué publié le 6 avril 2017, le Ministre de la Fonction Publique et de la Réforme Administrative a annoncé le recrutement spécial des auditeurs de justice et élèves greffiers d'expression anglaise. Ces enrôlements massifs s'inscrivent dans les mesures prises par le gouvernement pour amener les avocats anglophones à mettre un terme à un mouvement d'humeur qui dure depuis environ six mois. Le Chef de l'Etat, dans son discours de Bonne Année 2017 à la nation, apporta des éclairages sur les modalités qui règlent les revendications. La paix dans le pays doit être préservée. L'unité du pays doit être consolidée. Le Chef de l'Etat, Son Excellence Paul Biya a créé une Commission Nationale pour la Promotion du bilinguisme et du multiculturalisme. Le français et l'anglais sont les deux langues concernées par cette commission.

Aujourd'hui, je collabore avec des écoles de traducteurs et d'interprètes.

Actuellement, j’exerce dans la traduction technologique. En réali- 


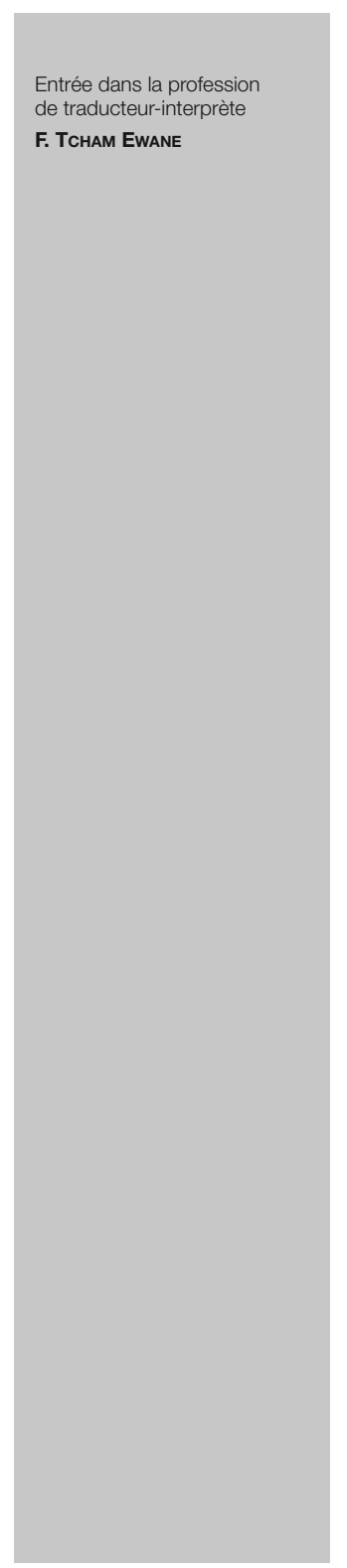

té, je dois aussi traduire des documents commerciaux et juridiques. Le français et l'italien sont mes deux langues pour la traduction.

On m'a proposé de m'inscrire à un master en traduction spécialisée, option juridique. L'interprète doit maîtriser la sémiotique, l'esthétique et aussi le journalisme.

Le traducteur-interprète est un pont entre deux groupes ou peuples qui ne parlent pas la même langue. Ce profil est très recherché dans des projets de développement pilotés par des experts de langue différente. Le métier demande beaucoup d'abnégation et de spontanéité intellectuelle. 